\title{
Métodos alternativos para contagem de micro-organismos em carcaças suínas
}

\author{
Alternative methods for the enumeration of microorganisms in swine carcasses
}

\author{
Cristhiane Stecanella de Oliveira Cattani ${ }^{\mathrm{I}^{*}}$ Pedro Ivo Pinheiro Fuchs ${ }^{\mathrm{I}}$ Andréia Cirolini $^{\mathrm{I}}$ \\ Cleide Rosana Werneck Vieira ${ }^{\mathrm{I}}$
}

\section{RESUMO}

\begin{abstract}
Micro-organismos estão presentes em toda a cadeia de processamento da carne, desde a matéria-prima até o produto final e compete aos programas de qualidade industrial verificar se existe contaminação na matéria-prima e nos produtos finais para, dessa forma, garantir um produto seguro ao consumidor. Para tanto, faz-se necessário utilizar metodologias alternativas à convencional na rotina diagnóstica dos laboratórios para obtenção de resultados confíveis e em menor tempo possivel. Com objetivo de verificar e comparar a correlação e o tempo de processamento, da metodologia convencional com as metodologias alternativas do sistema Petrifilm ${ }^{T M}$ (3M) e sistema Tempo ${ }^{\circledR}$ (bioMeriéux), foi realizada contagem de micro-organismos mesófilos, de enterobactérias e de Escherichia coli. As amostras foram coletadas da superficie de carcaças suínas em um abatedouro com Inspeção Federal no Estado de Santa Catarina, Brasil. A contagem de micro-organismos mesófilos, de enterobactérias e Escherichia coli foi feita por meio de ensaios realizados com os métodos alternativos Tempo ${ }^{\circledR}$, Petrifilm ${ }^{\mathrm{TM}}$ e o método convencional de contagem de micro-organismos em placas. Após análise estatística, o coeficiente de correlação de Pearson ( $r$ ) demonstrou fortes correlações, acima de 0.70 entre as metodologias utilizadas para a contagem de enterobactérias e micro-organismos mesófilos, porém demonstrou moderada correlação, entre 0.30 a 0.70 , para o diagnóstico de Escherichia coli. O uso dos métodos alternativos testados em substituição à metodologia convencional pode ser utilizado para diagnóstico de Escherichia coli, enterobactérias e micro-organismos mesófilos, por haver concordancia entre os resultados encontrados, acrescido da rapidez dessas metodologias com beneficio direto para a indústria de carne suína.
\end{abstract}

Palavras-chave: métodos, carcaças, suínos, micro-organismos.

\section{ABSTRACT}

Micro-organisms are present throughout the meat processing chain, from raw materials until finished product.
Industrial quality programs check for contamination in raw materials and final products to ensure the safety of consumer products; for this reason, the use of alternative methods instead of the conventional methods used in routine diagnostic laboratories is necessary to obtain reliable results in the shortest time possible. Samples of pig carcasses were collected in a slaughterhouse with Federal Inspection in the State of Santa Catarina, Brazil. Mesophilic micro-organisms, Enterobacteriaceae and Escherichia coli were evaluated and tests performed by alternative methods, Tempo ${ }^{\circledR}$, Petrifilm ${ }^{\mathrm{TM}}$, and the conventional method of counting micro-organisms in Petri dishes. After statistical analysis, the Pearson correlation coefficient $(r)$ showed a strong correlation up to 0.70 between the methodologies used for the enumeration of Enterobacteriaceae and mesophilic microorganisms, show a moderate correlation between 0.30 to 0.70 , for the diagnosis of Escherichia coli. The use of alternative methods to replace the conventional method can be recommended for diagnosis of Escherichiacoli, Enterobacteriaceae and mesophilic micro-organisms, because there was consistency between the results and the speed was increased by the alternative methodologies benefiting the pork industry.

Key words: methods, swine carcasses, micro-organisms.

\section{INTRODUÇÃO}

A presença de micro-organismos na carne suína é o resultado da contaminação dos animais vivos, dos equipamentos, dos manipuladores e do ambiente de processamento(NESBAKKEN \& SKJERVE, 1996). Isso demostra a importância em se manejar os riscos associados à segurança da carne, desenvolvendo estratégias de controle dos patógenos em todas as etapas do processamento (SOFOS \&

IDepartamento de Ciência e Tecnologia de Alimentos, Centro de Ciências Agrárias, Núcleo de Microbiologia de Alimentos, Universidade Federal de Santa Catarina (UFSC), Rodovia Admar Gonzaga 1346, 88034-000, Itacorubi, Florianópolis, SC, Brasil. E-mail: cristhianecattani@gmail.com.*Autor para correspondência. 
GEORNARAS, 2010). Mostra também a necessidade em se utilizar metodologias para a detecção de micro-organismos de forma rápida e eficiente na rotina diagnóstica, com intuito de prover informação adequada da possível presença de patógenos na matéria-prima e nos produtos acabados no controle do processo de produção e para o monitoramento de práticas de higiene e limpeza industrial (BOER \& BEUMER, 1999).

Os métodos de detecção microbiológica são frequentemente categorizados em dois grupos: convencionais ou tradicionais e rápidos ou alternativos. Os métodos tradicionais caracterizam-se por ser laboriosos, empregarem grandes volumes de meios de cultivo e requererem um tempo considerável para a obtenção de análises e resultados (JASSON et al., 2010). Os métodos rápidos são alternativos aos convencionais e são projetados para obter o resultado final em menor tempo. Isso é altamente desejável na indústria de alimentos, apesar das técnicas serem mais caras e requererem pessoal com alto nível de treinamento (FORSYTHE, 2002).

Considerando-se que o Brasil é um grande exportador de carne suína e considerando a necessidade rápida de se conhecer a qualidade microbiológica desse produto, aliado à carência de pesquisas nessa matriz, esse estudo teve como objetivo verificar o tempo necessário para a execução das análises e a correlação entre a metodologia convencional e as metodologias alternativas do sistema Petrifilm ${ }^{\mathrm{TM}}$ (3M) e sistema Tempo $^{\circledR}$ (bioMeriéux), para o diagnóstico de Escherichia coli, enterobactérias e micro-organismos mesófilos na matriz de carne suína.

\section{MATERIAL E MÉTODOS}

Durante o período de março de 2010 a março de 2011, foram analisadas 152 carcaças suínas, coletadas no final da linha de um abatedouro sob Inspeção Federal e com abate diário de 4.500 suínos. Cada amostra foi constituída de swabs de esponja, colhidos em quatro pontos da carcaça (pernil, barriga, lombo e papada) e acondicionados em um único saco de stomacher estéril. Os quatro swabs de esponja juntos $\left(100 \mathrm{~cm}^{2}\right.$ cada) representam a amostragem de uma área correspondente a $400 \mathrm{~cm}^{2}$. As amostras das superfícies das carcaças foram coletadas pressionando-se a esponja, previamente umedecida em $100 \mathrm{~mL}$ de solução salina peptonada $0,1 \%$ estéril, sobre moldes delimitadores esterilizados, na área a ser amostrada $\left(100 \mathrm{~cm}^{2}\right)$, por cerca de 20 segundos, iniciando o procedimento no sentido vertical, depois esfregando horizontalmente e, por fim, no sentido diagonal. A esponja foi acondicionada em um saco de stomacher estéril e mantida sob refrigeração a $4^{\circ} \mathrm{C}$ até o momento da análise no laboratório, que não ultrapassou 24 horas da coleta. Para o preparo da amostra utilizada para todas as metodologias, inicialmente, procedeu-se uma fricção do saco de stomacher contendo a esponja em água peptonada $0,1 \%$ e, a partir desse homogeneizado, preparam-se diluições de 1:10 e 1:100 (BRASIL,2007).

$\mathrm{Na}$ metodologia convencional, a amostra homogeneizada e diluída em 1:10 e 1:100 foi inoculada em ágar (Oxóid) específico para cada tipo de micro-organismo pesquisado, seguindo o preconizado para a metodologia convencional para os micro-organismos mesófilos, enterobactérias e Escherichia coli (MORTON, 2001; KORNACKI \& JOHNSON, 2001).

A metodologia alternativa Petrifilm $^{\mathrm{TM}}$ é um sistema de plaqueamento chamado all-in-one, no qual a placa de Petrifilm ${ }^{\mathrm{TM}}$ faz uso de um fino filme plástico que carrega o meio de cultura (JASSON et al., 2010). Os ingredientes desse meio de cultura variam para cada tipo de micro-organismo a ser identificado. Para a contagem de micro-organismos mesófilos neste sistema, inoculou-se $1 \mathrm{~mL}$ do homogeneizado e das diluições 1:10 e 1:100 em cartões Petrifilm ${ }^{\mathrm{TM}} \mathrm{AC}$ e incubou-se $36^{\circ} \mathrm{C} \pm 1^{\circ} \mathrm{C}$ por 48 horas. Para contagem de enterobactérias, inoculou-se $1 \mathrm{~mL}$ do homogeneizado e das diluições 1:10 e 1:100 em cartões Petrifilm ${ }^{\mathrm{TM}}$ EB e incubou-se a $36^{\circ} \mathrm{C} \pm 1^{\circ} \mathrm{C}$ por 24 horas. Para a contagem de Escherichia coli, inoculou-se $1 \mathrm{~mL}$ do homogeneizado e das diluições 1:10 e 1:100 sobre cartões Petrifilm ${ }^{\mathrm{TM}} \mathrm{EC}$ e incubou-se a $36^{\circ} \mathrm{C} \pm 1^{\circ} \mathrm{C}$ por 24 horas.Após a incubação, procedeu-se à contagem das colônias e o resultado foi registrado em $\mathrm{UFCcm}^{-2}$ (KORNACKI \& JOHNSON, 2001).

$\mathrm{Na}$ metodologia utilizada para o sistema Tempo ${ }^{\circledR}$, para contagem total de micro-organismos mesófilos (TVC), reconstituiu-se o meio TVC com 3,0mL de água destilada esterilizada. Em seguida, adicionou-se $1 \mathrm{~mL}$ da amostra homogeneizada preparada sem diluição, completando $4 \mathrm{~mL}$ de volume total. Para a contagem de enterobactérias (EB), reconstituiu-se o meio $\mathrm{EB}$ com $3,9 \mathrm{~mL}$ de água destilada esterilizada, adicionando $0,1 \mathrm{~mL}$ da amostra homogeneizada, preparada sem diluição. Para a contagem de Escherichia coli (EC), reconstituiu-se o meio EC com 3,9mL de água destilada esterilizada e, em seguida, adicionou-se $0,1 \mathrm{~mL}$ da amostra homogeneizada. Os frascos e os cartões foram colocados no interior do equipamento, em que ocorreu a transferência do conteúdo do frasco para o cartão que contém 48 poços com três diferentes 
volumes $(16 \times 225 \mu \mathrm{L} ; 16 \times 22,5 \mu \mathrm{L} ; 16 \times 2,25 \mu \mathrm{L})$. Imediatamente após o enchimento, os cartões foram selados. Essa operação ocorreu no interior da estação de trabalho denominada Tempo ${ }^{\circledR}$ preparo. Os cartões foram incubados em estufa por 24 horas, a $35^{\circ} \mathrm{C}$ para EB e EC e, por 48 horas, a $35^{\circ} \mathrm{C}$ para TVC. Após esse período, realizou-se a leitura dos cartões na estação Tempo $^{\circledR}$ leitora. Uma vez que a leitura foi concluída, os resultados foram analisados automaticamente pelo sistema, que determina quais os poços que foram positivos, gerando os resultados em UFC $\mathrm{cm}^{-2}$.

Foi utilizada a análise de regressão linear na análise estatística dos dados, determinando-se o coeficiente de correlação linear de Pearson (r), que varia de -1 a 1, para quantificar a correlação entre as metodologias utilizadas. Nesse intervalo, os valores foram distribuídos, sendo considerado 0 para inexistência de correlação, 0,30 a 0,70 moderada correlação e de 0,70 a 1 forte correlação, não sendo encontrado nenhum resultado de correlação negativa $(-1)$. Os dados referentes aos micro-organismos pesquisados foram analisados estatisticamente pelo programa STATÍSTICA 7.0, sendo realizada Análise de variância e teste de Tukey, em nível de $95 \%$ de confiança (ANDRADE \& OGLIARI, 2007).

\section{RESULTADOS E DISCUSSÃO}

Neste estudo, pode-se observar a redução do tempo de execução entre as metodologias utilizadas. Para as enterobactérias, a redução do tempo desde o preparo da amostra até o resultado final emitido pelo sistema Petrifilm ${ }^{\mathrm{TM}}$ e pelo sistema Tempo $^{\circledR}$ foi de 24 horas em relação à metodologia convencional. Para a contagem de Escherichia coli, houve uma redução de 144 horas dos dois sistemas alternativos em relação à metodologia convencional.

Através dos resultados da contagem de micro-organismos mesófilos, enterobactérias e Escherichia coli, é possível observar que houve concordância entre as diferentes metodologias utilizadas, ao se examinar o coeficiente de correlação (r) em que consta uma associação linear positiva entre as metodologias, conforme apresentado na tabela 1, concordando com coeficientes encontrados pelos autores CROWLEYet al. (2009), SOHIER et al. (2007) e OWEN et al. (2010).

Com relação aos micro-organismos mesófilos, pode ser verificado que estes foram detectados em todas as metodologias utilizadas. Os resultados médios de contagem foram semelhantes entre as metodologias, sem diferença estatística entre as médias, sendo encontrado $3,44 \log _{10}$ de microorganismos mesófilos para o método convencional, $3,47 \log _{10}$ de micro-organismos mesófilos para o Petrifilm $^{\mathrm{TM}}$ e de $3,34 \log _{10}$ de micro-organismos mesófilos para o Tempo ${ }^{\circledR}$, com um desvio padrão de 0,$66 ; 0,63$; e 0,66 , respectivamente.

Escherichia coli foi detectada em todos os métodos utilizados. A presença da Escherichia coli no Tempo ${ }^{\circledR}$ foi de $32,89 \%$ (50/152), no Petrifilm ${ }^{\mathrm{TM}}$ foi de $38,82 \%$ (59/152) e no método convencional foi de $31,58 \%$ (48/152). As enterobactérias foram detectadas em todos os métodos, sendo de 44,08\% (67/152) no Tempo $^{\circledR}$, de $61,84 \%(94 / 152)$ no Petrifilm ${ }^{\mathrm{TM}}$, e de $19,08 \%$ (29/152) no método convencional. Não houve diferença significativa entre as médias dos micro-organismos mesófilos, de Escherichia coli e de enterobactérias, nos métodos testados.

CATTAPAN et al. (2007), JOHNSON (2007), KUNICKA (2007) e SOHIER et al. (2007) verificaram a redução de tempo em estudos de fluxo de trabalho, que demonstraram uma redução de duas a três vezes no tempo de manipulação da amostra com a utilização do Tempo $^{\circledR}$, em comparação com o método de referência ISO. Esses autores relatam a redução e a otimização do tempo de ensaio das amostras, que se deve ao tipo de sistema utilizado pelo equipamento Tempo ${ }^{\circledR}$, totalmente automatizado e baseado no metabolismo de substratos específicos produzidos pelos micro-organismos. Esse sistema miniaturizado utiliza o conceito da placa microtituladora, que permite reduzir o volume reativo e o meio a ser empregado nos ensaios. A otimização do

Tabela 1 - Coeficiente de correlação (r) entre as diferentes metodologias (Convencional, Petrifilm ${ }^{\mathrm{TM}}$ e Tempo $^{\mathrm{B}}$ ) na contagem de microorganismos mesófilos, enterobactérias e Escherichia coli da superfície de carcaças suínas no período de março de 2010 a março de 2011.

\begin{tabular}{|c|c|c|c|}
\hline Metodologias & Mesófilos & Enterobactérias & Escherichia coli \\
\hline Convencional $\mathrm{x}$ Tempo ${ }^{\circledR}$ & $\mathrm{r}=0,6712$ & $\mathrm{r}=0,7535$ & $\mathrm{r}=0,6255$ \\
\hline Convencional $\times$ Petrifilm ${ }^{\mathrm{TM}}$ & $\mathrm{r}=0,7770$ & $\mathrm{r}=0,8368$ & $\mathrm{r}=0,3955$ \\
\hline Petrifilm $^{\mathrm{TM}} \times$ Tempo $^{\circledR}$ & $\mathrm{r}=0,8118$ & $\mathrm{r}=0,7480$ & $\mathrm{r}=0,5379$ \\
\hline
\end{tabular}

Legenda: $r=$ coeficiente de correlação de Pearson.

Ciência Rural, v.43, n.6, jun, 2013. 
tempo e a especificidade do ensaio, utilizando-se a metodologia do sistema Tempo $^{\circledR}$ também pode ser verificada nos resultados deste estudo.

A redução do tempo de ensaio e resposta pelo sistema Petrifilm ${ }^{\mathrm{TM}}$ se deve à simplicidade de manipulação e rapidez na contagem . O processo de identificação dos micro-organismos é realizado utilizando sais de tetrazólio como indicadores. Esse processo é considerado um método alternativo indireto para medir atividade respiratória do micro-organismo. A redução desse sal pela enzima mitocondrial succinato-desidrogenase leva à formação de um precipitado insolúvel de cor intensa. Os sais de tetrazólium, o cloreto de trifeniltetrazolium (TTC) e o cloreto de iodo-nitrofeniltetrazólium (INT) utilizados são rapidamente reduzidos pela maioria dos sistemas desidrogenases dos micro-organismos. A transformação realiza-se somente em células vivas e a quantidade do composto produzido é proporcional ao número de células presentes. SILVA et al. (2006) recomendam o uso de Petrifilm ${ }^{\mathrm{TM}}$ EC como metodologia sensível e eficiente para contagem de Escherichia coli em alimentos de origem animal, em substituição à metodologia convencional. A simplicidade e rapidez dessa metodologia puderam ser verificadas nos ensaios deste estudo, realizados com a metodologia do sistema Petrifilm ${ }^{\mathrm{TM}}$.

Houve uma moderada correlação entre a metodologia convencional e o Tempo $^{\circledR}(\mathrm{r}=0,62)$ para a Escherichia coli. Os resultados ainda demonstram uma moderada correlação entre a metodologia convencional e o Petrifilm ${ }^{\mathrm{TM}}(\mathrm{r}=0,39)$ e entre as duas metodologias alternativas Tempo ${ }^{\circledR}$ e Petrifilm ${ }^{\text {TM }}(\mathrm{r}=0,53)$. A concordância entre a metodologia convencional e o Tempo ${ }^{\circledR}$ para detecção de Escherichia coli neste estudo foi menor que a concordância encontrada nos ensaios realizados por GREEN et al. (2007), sendo $r=0,92$ entre o método Tempo $^{\circledR}$ e a metodologia convencional em placa para avaliação de Escherichia coli em amostras de produtos cárneos bovinos e de aves. A correlação também foi menor que a encontrada nos estudos comparativos realizados por DEVULDER et al. (2007), r=0,99 em produtos a base de carne. Podemos atribuir as diferenças de correlação dos diferentes estudos na detecção da Escherichia coli, à qualidade microbiológica das amostras.

Estudos realizados por SOHIER et al. (2007) demonstram níveis similares ou mais altos de repetibilidade e reprodutibilidade pelo Tempo ${ }^{\circledR}$, comparados com o método padrão de contagem em placas. CROWLEY et al. (2010) mostram em seu trabalho que, para a detecção de Escherichia coli, o
Tempo ${ }^{\circledR}$ EC possui, no meio de cultura, o substrato fluorogênico, 4-metilumbelliferil- $\beta$-D-glucuronideo (MUG), que é clivado pela enzima $\beta$-glucoronidase, produzida pela maioria das cepas da Escherichia coli. Ao ser clivado, o MUG produz fluorescência, sendo então detectada pelo Tempo ${ }^{\circledR}$ Reader. Dentre os métodos utilizados neste estudo, a melhor concordância para detecção de Escherichiacoli $(\mathrm{r}=0,62)$ foi verificada entre a metodologia convencional e o Tempo $^{\circledR}$, o que demonstra a sensibilidade e especificidade de detecção do método, confirmada pelos resultados encontrados neste estudo.

Para as enterobactérias, houve forte correlação entre a metodologia convencional e o Tempo $^{\circledR}(\mathrm{r}=0,75)$, entre a metodologia convencional e o Petrifilm ${ }^{\text {TM }}(\mathrm{r}=0,83)$ e entre as duas metodologias alternativas Tempo e Petrifilm ${ }^{\mathrm{TM}} \quad(\mathrm{r}=0,75)$. Em estudo realizado por OWEN et al. (2010), foram encontrados coeficientes de correlação equivalentes $(\mathrm{r}=0,75$ a 0,78$)$ aos encontrados neste estudo, quando avaliou o Tempo $^{\circledR}$ EB em relação à técnica do número mais provável e semeadura em superfície e profundidade. A detecção de enterobactérias pelo Tempo $^{\circledR}$ foi demonstrada em estudo realizado por SOHIER et al. (2007), com níveis similares ou mais altos de repetibilidade e reprodutibilidade desse método, quando comparado com o método padrão de contagem em placas.

A especificidade do Tempo $^{\circledR}$ EB para detecção de enterobactérias pode ser entendida pela reação que ocorre entre o micro-organismo e o meio de cultura presente nos frascos que contêm uma molécula fluorescente, o 4-metil umbelliferone (4MU), indicadora de pH (JOHNSON et al., 2008). Quando o pH está neutro, há emissão de fluorescência. Os micro-organismos da família Enterobacteriaceae presentes no cartão assimilam os nutrientes do meio de cultura durante a incubação, resultando em um incremento do $\mathrm{pH}$. Quando ocorre a fermentação da glicose por esses micro-organismos, há acidificação do reagente, que resulta em extinção da fluorescência em tubos com reação positiva, conforme já relatado em estudos realizados por OWEN et al.(2010), JOHNSON et al. (2009), COLÓN-REVELES et al.(2008) e SILBERNAGEL \& LINDBERGER (2003). Esses pesquisadores obtiveram precisão nos resultados em estudo de repetitividade, em diversas amostras de alimentos com o uso do Petrifilm ${ }^{\mathrm{TM}} \mathrm{e}$ a metodologia convencional, e recomendam o seu uso na contagem de enterobactérias para diversos alimentos, entre eles, carne preparada congelada. Nesta pesquisa, foram obtidos resultados positivos com $r=0,83$ de concordância entre o Petrifilm ${ }^{\mathrm{TM}}$ EB 
e a metodologia convencional para enterobactérias em swab de carcaça suína, concordando com o estudo feito por SILBERNAGEL \& LINDBERGER (2002), no qual o Petrifilm ${ }^{\mathrm{TM}} \mathrm{EB}$ foi mais sensível e seletivo, se comparado à sensibilidade do método convencional em placa com ágar Violeta Vermelho Bile Glicose (VRBG). Ambos os métodos alternativos utilizados neste estudo foram sensíveis e específicos na detecção das enterobactérias.

A correlação foi mediana entre a metodologia convencional e o Tempo ${ }^{\circledR}(\mathrm{R}=0,67)$ para os micro-organismos mesófilos. Esses resultados representam menor correlação em relação ao estudo de avaliação realizado por GREEN et al. (2007), quando foi encontrado $\mathrm{r}=0,97$ entre o método Tempo ${ }^{\circledR}$ e o método convencional em placa para a contagem de micro-organismos mesófilos. O mesmo resultado foi observado em relação ao estudo realizado por DEVULDER et al. (2007) e CROWLEY et al. (2009), que encontraram $\mathrm{r}=0,95$ de concordância para as mesmas metodologias. Foi observada, neste estudo, uma forte correlação entre a metodologia convencional e o Petrifilm ${ }^{\mathrm{TM}}(\mathrm{R}=0,77)$ e entre as duas metodologias alternativas Tempo ${ }^{\circledR}$ e Petrifilm ${ }^{\mathrm{TM}}$ $(\mathrm{R}=0,81)$.

\section{CONCLUSÃO}

Embora algumas pesquisas com os métodos alternativos estudados já tenham sido publicadas, os resultados deste estudo contribuíram para a avaliação dessas metodologias em matriz de carnes suínas até então não estudadas.

Neste estudo, as amostras foram provenientes de $s w a b$ de carcaças suínas processadas em um abatedouro sob Inspeção Federal, com todos os programas de Boas Práticas de Fabricação e qualidade implantadas, o que conferiu qualidade microbiológica às amostras e baixo número de Escherichia coli detectadas nas metodologias avaliadas.

A metodologia do sistema TEMPO $^{\circledR}$ demonstrou vantagem em relação às demais metodologias avaliadas neste estudo, por processar um número maior de amostras por intervalo de tempo, reduzir o volume de material e espaço necessário para realização dos ensaios e pela automatização que confere rapidez ao processo, porém a metodologia do sistema Petrifilm ${ }^{\mathrm{TM}}$ mostrou resposta ligeiramente melhor com relação à contagem de micro-organismos mesófilos e enterobactérias. Ambos os métodos podem ser recomendados para a matriz de carne suína, beneficiando assim a indústria processadora que necessita de respostas rápidas no seu processo produtivo.

\section{REFERÊNCIAS}

ANDRADE, D.F.; OGLIARI, P.J. Estatística para as ciências agrárias e biológicas. Florianópolis: UFSC, 2010. 470p.

BOER, E.D.; BEUMER, R.R. Methodology for detection and typing of foodborne microorganisms. International Journal of Food Microbiology, v.50, p.119-130, 1999. Disponível em: <http:// www.sciencedirect.com/science/article/pii/S0168160599000811>. Acesso em: 10 jun. 2011. doi:S0168-1605(99)00081-1.

BRASIL. Ministério da Agricultura Pecuária e Abastecimento. Circular n.130 de 13 de fevereiro de 2007. Brasília, 2007. Disponível em: <http://sigsif.agricultura.gov.br/sigsif/principal_ sigsif $>$. Acesso em: 11 jun. 2011.

CATTAPAN, F. et al. New approach for workflow evaluation in food microbiology laboratories: $\mathrm{TEMPO}^{\circledR}$, Automated MPN Method Versus Plate Count Method. bioMérieux, France, 2007. Acesso em: 05 maio 2011. Online. Disponível em: $<$ http://www.foodprotection.org/files/annual_meeting/posterabstracts-2007.pdf $>$.

CROWLEY, E.S. et al. TEMPO ${ }^{\circledR}$ TVC for the enumeration of aerobic mesophilic flora in foods: collaborative study. Journal of AOAC International, v.92, p.165-174, 2009. Disponível em: <http://www.foodhygiene.or.kr/admin/down_file.php.pdf $>$. Acesso em: 05 maio, 2011.

CROWLEY, E.S. et al. TEMPO ${ }^{\circledR} \mathrm{EC}$ for the enumeration of Escherichia coli in foods: collaborative study. Journal of AOAC International, v.93, p.576-586, 2010. Disponível em: <http:// www.ncbi.nlm.nih.gov/pubmed.> Acesso em: 05 maio, 2011.

COLÓN-REVELES, J. et al. Evaluation of the TEMPO ${ }^{\circledR}$ EB method for the enumeration of Enterobacteriaceae in foods. IAFP, p.3-47, 2008. Disponível em: <http://www.biomerieuxusa. com/upload/TEMPO $\% 20 E B \% 20 b M x \% 20 U S-2 . p d f>$. Acesso em: 02 maio, 2011.

DEVULDER, G. et al. Comparison of TEMPO ${ }^{\circledR}$ TVC, TEMPO ${ }^{\circledR}$ TC, TEMPO ${ }^{\circledR}$ EC with conventional culture methods in Meat Based Products. bioMérieux, France, 2007. Acesso em:02 maio, 2011. Online. Disponível em: <http://www.biomerieux-usa.com/ upload/TEMPO.pdf $>$.

FORSYTHE, S.J. Microbiologia da segurança alimentar. Porto Alegre: Artmed, 2002. 424p.

GREEN, R. et al. Evaluation of the TEMPO ${ }^{\circledR}$ System for total viable count, total coliforms and $\boldsymbol{E}$. coli enumeration in meat and poultry products. bioMérieux, France, 2007. Acesso em: 02 maio, 2011. Online. Disponível em: $<$ http://www.biomerieuxusa. com/upload/TVC.pdf $>$.

JASSON, V. et al. Alternative microbial methods: an overview and selection criteria. Food Microbiology, v.27, p.710-730, 2010. Disponível em: <http://www.sciencedirect.com/science/article/pii/ S0740002010000821>. Acesso em: 10 jun. 2011. doi: 10.1016/j. fm.20120.04.008

JOHNSON, R. BioMérieux TEMPO ${ }^{\circledR}$ EC test granted PTM status. Inside laboratory management. AOAC International, 2007. Acesso em: 02 maio, 2011. Online. Disponível em: $<$ http://www. biomerieuxusa.com/upload/EC\%20ILM\%20FINAL\%20May. June\%202007-1.pdf>. 
JOHNSON, R. et al. BioMérieux TEMPO ${ }^{\circledR}$ TVC test granted PTM status. Insidelaboratory management. AOAC International, 2008. Acesso em: 02 maio, 2011. Online. Disponível em: $<$ http:// www.biomerieuxusa.com/upload/TVC.pdf>.

JOHNSON, R. et al. BioMérieux TEMPO ${ }^{\circledR}$ EB test granted PTM status. Inside laboratory management. AOAC International, 2009. Acesso em: 02 maio, 2011. Online. Disponível em: $<$ http:// www.biomerieuxusa.com/upload/EB.pdf>.

KORNACKI J.L.; JOHNSON J.L. Enterobacteriaceae Coliforms, and Escherichia coli as quality and safety indicators. In: . Compendium of methods for the microbiological examination of foods. 4.ed. Washington DC: American Public Health Association, 2001. Cap.8, p.69-82.

KUNICKA, A. Evaluation of the TEMPO ${ }^{\circledR}$ SYSTEM: an automated method for food microbiological quality control. Journal of Biotechnology, v.131, p.69-72, 2007. Disponível em:<http://www.sciencedirect.com/science/article/pii/ S0168165607005792>. Acesso em: 08 jun. 2011. doi:10.1016/j. jbiotec2007.07.119.

MORTON, R.D. Aerobic plate count. In Compendium of methods for the microbiological examination of foods. 4.ed. Washington DC: American Public Health Association, 2001. Cap.7, p.63-67.

NESBAKKEN, T;; SKJERVE, E. Interruption of microbial cycles in farm animals from farm to table. Meat Science, v.43, p.S47-S57, 1996. Disponível em: <http://www.sciencedirect. com/science/article/pii/030917409600054X>. Acesso em: 03 jun. 2011. doi:S0309-1740(96)0054-X.

OWEN, M. et al. Evaluation of the TEMPO ${ }^{\circledR}$ most probable number technique for the enumeration of Enterobacteriaceae in food and dairy products. Journal of Applied Microbiology, v.109, p.1810-1816, 2010. Disponível em: <http://www.ncbi. nlm.nih.gov/pubmed/20649835>. Acesso em: 07 jun. 2011. doi:10.1111/j.1305-2672.2010.04810.x.

SILBERNAGEL, K.; LINDBERG, K.G. $3 \mathrm{M}^{\mathrm{TM}}$ Petrifilm $^{\mathrm{TM}}$ Enterobacteriaceae count plate method for enumeration of enterobacteriaceae in selected foods: collaborative study. Journal of AOAC international, v.86, n.4, p.802-815, 2003. Acesso em: 05 maio, 2012. Online. Disponível em: <http://www.3m.com/ intl $/ \mathrm{kr} / \mathrm{microbiology} / \mathrm{regu}$ info/3.pdf $>$.

SILBERNAGEL, K.M.; LINDBERG, K.G. Evaluation of the 3M Petrifilm Enterobacteriaceae count plate method for the Enumeration of Enterobacteriaceae in foods. Journal of Food Protection, v.65, p.1452-1456, 2002. Disponível em: <http:// www.3m.com $/ \mathrm{intl} / \mathrm{kr} / \mathrm{microbiology} / \mathrm{regu}$ info/3.pdf $>$. Acesso em: 06 maio, 2012.

SILVA, M.P. et al. Avaliação do padrão coliformes a $45^{\circ} \mathrm{C}$ e comparação da eficiência das técnicas dos tubos múltiplos e Petrifilm EC na detecção de coliformes totais e Escherichia coli em alimentos. Ciência Tecnologia Alimentar, v.26, p.352-359, 2006. Disponível em: $<$ http://www.scielo.br/scielo.php?script=sci_arttex t\&pid=S0101-20612006000200018>. Acesso em: 03 jun. 2012. doi:10.1590/S0101-20612006000200018.

SOFOS, J.N.; GEORNARAS, I. Overview of current meat hygiene and safety risks and summary of recent studies on biofilms, and control of Escherichia coli O157:H7 in nonintact, and Listeria monocytogenesin ready-to-eat, meat products. Meat Science, v.86, p.2-14, 2010. Disponível em: <http://www.sciencedirect. com/science/article/pii/S0309174010001476>. Acesso em: 03 jun. 2011. doi:10.1016/j.meatsci.2010.04.015.

SOHIER, D. et al. TEMPO ${ }^{\circledR}$ system :AFNOR validation according to the ISO 16140 standard 2006. bioMérieux, France, 2007. Acesso em: 02 maio, 2011. Online. Disponível em: <http://www. biomerieuxusa.com/upload/ISO $\% 2016140 \% 20$ validation $\% 20$ by $\% 20$ AFNOR $\% 20$ on $\% 20$ TVC, $\% 20$ TC $\% 20$ and $\% 20$ EC, $\% 20$ Adria-2.pdf>. 